\title{
Computational Studies on Nonenzymatic and Enzymatic Pyridoxal Phosphate Catalyzed Decarboxylations of 2-Aminoisobutyrate ${ }^{\dagger}$
}

\author{
Michael D. Toney* \\ Department of Chemistry, University of California, One Shields Avenue, Davis, California 95616
}

Received May 31, 2000; Revised Manuscript Received August 30, 2000

\begin{abstract}
A computational study of nonenzymatic and enzymatic pyridoxal phosphate-catalyzed decarboxylation of 2-aminoisobutyrate (AIB) is presented. Four prototropic isomers of a model aldimine between AIB and $5^{\prime}$-deoxypyridoxal, with acetate interacting with the pyridine nitrogen, were employed in calculations of both gas phase and water model (PM3 and PM3-SM3) decarboxylation reaction paths. Calculations employing the transition state structures obtained for the four isomers allow the demonstration of stereoelectronic effects in transition state stabilization as well as a separation of the contributions of the Schiff base and pyridine ring moieties to this stabilization. The unprotonated Schiff base contribution $(\sim 16 \mathrm{kcal} / \mathrm{mol})$ is larger than that of the pyridine ring even when it is protonated $(\sim 10 \mathrm{kcal} / \mathrm{mol})$, providing an explanation of the catalytic power of pyruvoyl-dependent amino acid decarboxylases. An active site model of dialkylglycine decarboxylase was constructed and validated, and enzymatic decarboxylation reaction paths were calculated. The reaction coordinate is shown to be complex, with proton transfer from Lys272 to the coenzyme $\mathrm{C}^{\prime}$ likely simultaneous with $\mathrm{C} \alpha-\mathrm{CO}_{2}{ }^{-}$bond cleavage. The proposed concerted decarboxylation/proton-transfer mechanism provides a simple explanation for the observed specificity of this enzyme toward oxidative decarboxylation.
\end{abstract}

Pyridoxal phosphate (PLP) ${ }^{1}$ dependent enzymes are ubiquitous and central to a wide variety of anabolic and catabolic pathways in the metabolism of nitrogen-containing compounds. The first, obligatory chemical step in all PLP dependent enzymatic reactions is formation of a Schiff base between the coenzyme aldehydic and substrate amino groups (Scheme 1). This common intermediate is termed the "external aldimine" in the literature. The general utility of PLP stems from its ability to stabilize carbanions formed adjacent to the Schiff base in the external aldimine intermediate through delocalization into the extended $\pi$ bonded system (i.e., Schiff base and pyridine ring).

Dunathan (32) proposed that the common fundamental mechanism that PLP dependent enzymes use to control reaction specificity is specific binding of the external aldimine intermediate such that the bond to be broken is oriented parallel to the $p$ orbitals of the $\pi$-bonded system. This orientation maximizes orbital overlap between the nascent $p$ orbital on $\mathrm{C} \alpha$ and the $\pi$ system in the transition state, thereby minimizing transition state energy. The magnitude of these proposed stereoelectronic effects have never been addressed.

Dialkylglycine decarboxylase (DGD) is an intriguing PLP dependent enzyme that catalyzes two mechanistically different reactions: oxidative decarboxylation of 2,2-dialkylgycines in the first half-reaction, and transamination of $\alpha$-keto acids in the second. Several DGD structures have been solved by X-ray crystallography (1-4).

\footnotetext{
' Supported by Grant GM54779 from the U.S. National Institutes of Health.

* To whom correspondence should be addressed. Phone: (530) 7520655. Fax: (530) 752-8995. E-mail: toney@chem.ucdavis.edu.

${ }^{1}$ Abbreviations: PLP, pyridoxal 5'-phosphate; DGD, dialkylglycine decarboxylase; AIB, 2-aminoisobutyrate.
}

The active site structure shows that, to be bound productively for decarboxylation, a carboxylate group would make multiple favorable hydrogen bonding and electrostatic interactions with side chains of active site residues (3), counter to expectation since charge neutralization reactions occur more readily in nonpolar vs polar environments (5). This assumes that the substrate carboxylate group and active-site side-chain functional groups are ionized, as found spectroscopically for functional groups in other enzymes $(6-18)$. The pyruvoyl dependent histidine decarboxylase does place its substrate carboxylate in a nonpolar environment (19), and nonpolar binding sites were successfully employed in the generation of PLP-dependent catalytic antibodies (20).

Enzymologists are beginning to apply productively the methods of computational chemistry to the study of enzyme catalyzed reactions $(21-23)$. This report describes the use of the PM3 semiempirical method to calculate decarboxylation reaction paths for a model AIB-PLP aldimine, to quantitate stereoelectronic effects on this reaction, and to provide a separation of the contributions of the Schiff base and pyridine ring. An active-site model of DGD is also employed in decarboxylation reaction path calculations. The latter suggest that the observed strict specificity for oxidative decarboxylation of AIB is achieved by a concerted decarboxylation/proton-transfer mechanism, as suggested in the preceding paper in this issue (24).

\section{MATERIALS AND METHODS}

Version 5.0 of the program SPARTAN (Wavefunction, Inc.) was used for all calculations. This version of SPARTAN incorporates the MMFF94 force field, which was used in all mechanics calculations. All PM3 calculations used the standard, unmodified parameters supplied, and restricted 
Scheme 1

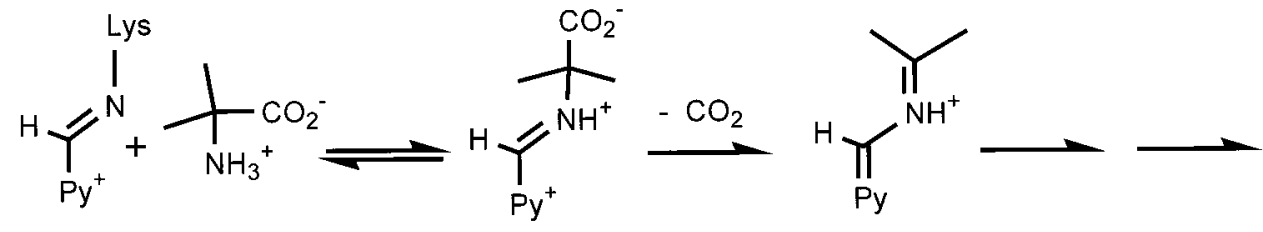

Internal Aldimine

External Aldimine

Quinonoid

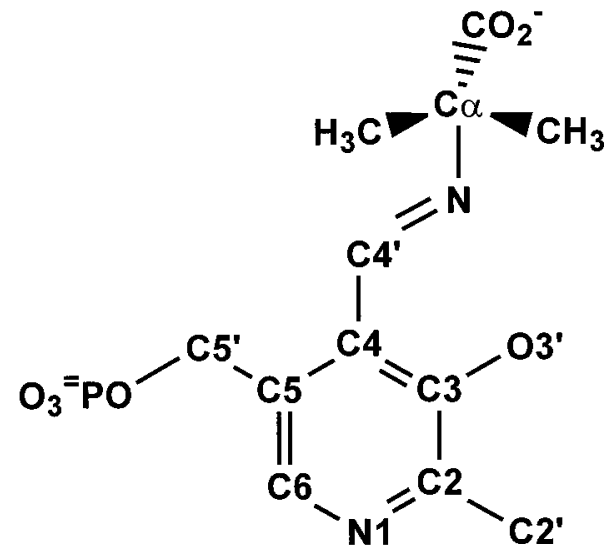

Hartree-Fock wave functions. SPARTAN has the advantage for protein-oriented calculations that atomic positions can easily be frozen in Cartesian coordinates. It has the disadvantage that the number of atoms is limited to 200 for semiempirical calculations. This limit, rather than computational resources, restricted the size of the current activesite model.

An active site model whose coordinates were taken from the structure of the most active form of the enzyme $\left(\mathrm{K}^{+}\right.$liganded form) was constructed in INSIGHTII, under the 200 atom constraint of the semiempirical module of SPARTAN. This model is shown in Figure 4A. In most cases, the protein backbone atoms were removed, and the $\mathrm{C} \alpha$ atoms replaced with hydrogens. For Gln52, the side chain was truncated through the $\mathrm{C} \beta$ atom since this residue is held tightly in place in the protein structure by two hydrogen bonds to the amide oxygen atom. The phosphate group of the coenzyme was replaced by a methoxy group, since the former plays no direct role in the catalytic mechanism as surmised from the X-ray structure $(1,3)$. The atoms that were held fixed in all the calculations reported here are shown rendered as spheres in Figure 4A. In general, the $\mathrm{C} \beta$ 's, and the hydrogens corresponding to the $\mathrm{C} \alpha$ 's, were fixed. The amide oxygen of Gln52 was fixed, as were $\mathrm{C} \beta$ and $\mathrm{C} \gamma$ of Asp243 since these atoms are held firmly in place by the enzyme structure. To reiterate for emphasis, only the $\mathrm{C} \beta$ 's and hydrogens corresponding to C $\alpha$ 's, and atoms firmly locked by multiple hydrogen bonds in the protein structure, were held fixed. The AIB substrate was built as a Schiff base with the coenzyme by analogy to the structures of inhibitor-bound DGD. Lys272, which forms a Schiff base with the coenzyme in the unliganded structure, was initially built with a protonated amino group interacting with the AIB carboxylate.

Metropolis Monte Carlo calculations as implemented in SPARTAN were applied to the active-site model with the AIB Schiff base in order to eliminate any potential biases in model building. All of the nonring carbon-carbon bonds
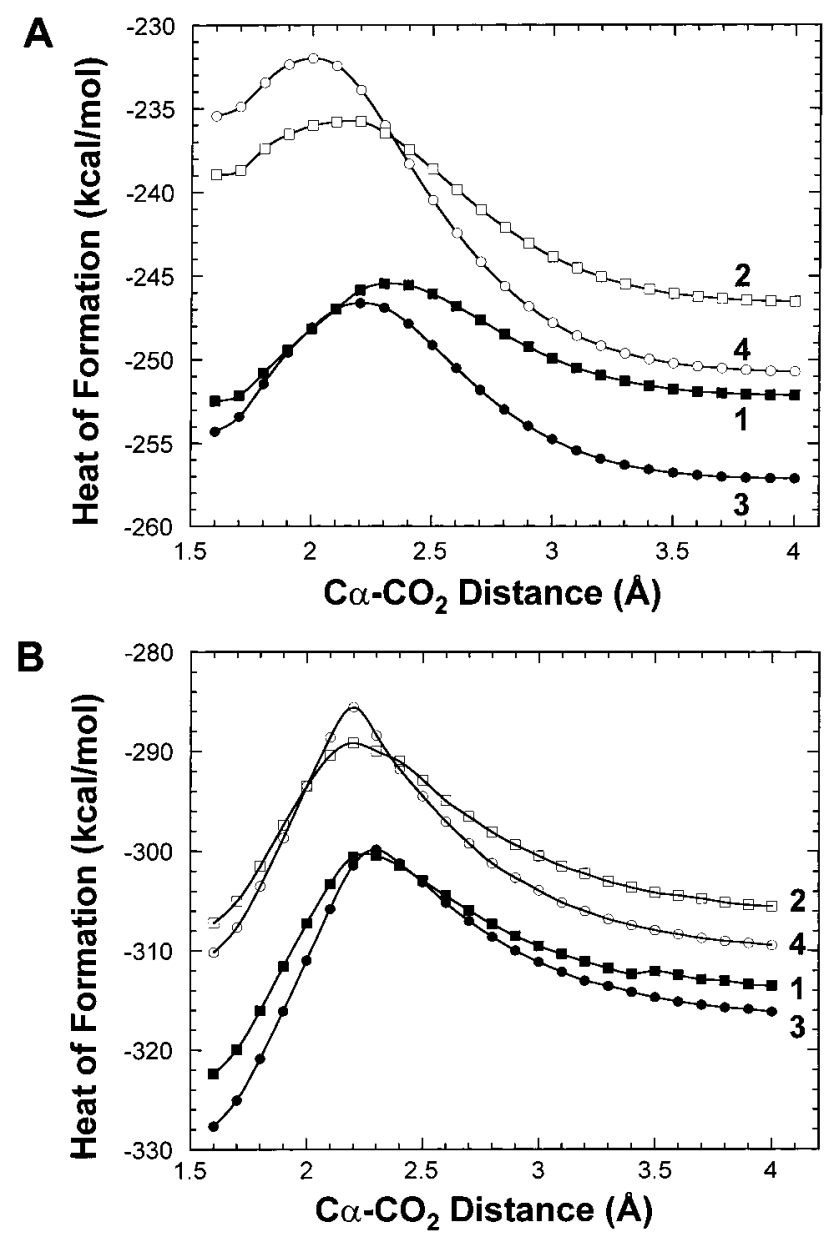

FIGURE 1: PM3 decarboxylation reaction path calculations for the four isomers of the simple aldimine model shown in Scheme 2. Constrained energy optimizations were performed (A) in the gas phase and (B) in the presence of the SM3 water model.

were allowed to rotate with $60^{\circ}$ resolution. A maximum of five bonds was allowed to rotate in the generation of a given 


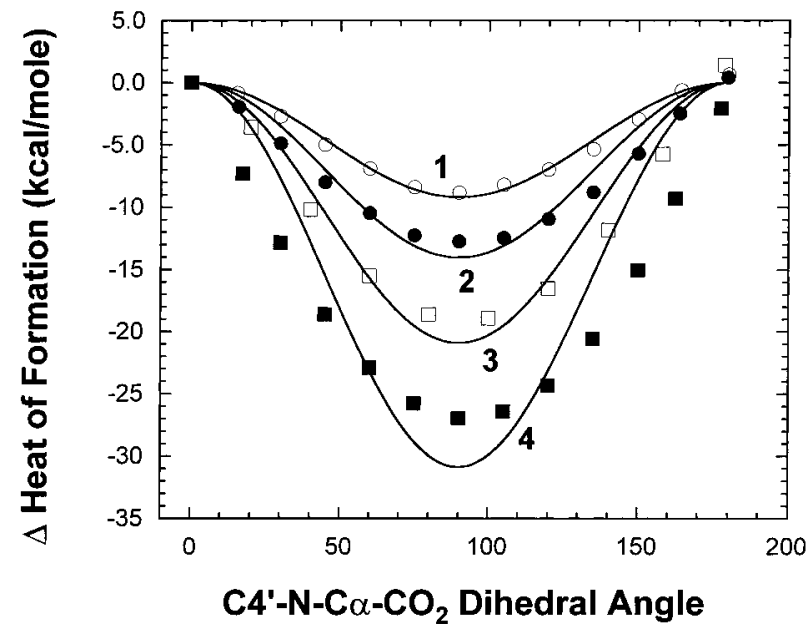

FIGURE 2: PM3 coordinate driving calculations to determine the total resonance stabilization of the transition state, and its geometric dependence. The $\mathrm{C} \alpha-\mathrm{CO}_{2}{ }^{-}$bond lengths were fixed at the transition state values. The $\mathrm{C} 4^{\prime}-\mathrm{N}-\mathrm{C} \alpha-\mathrm{CO}_{2}$ dihedral angle was then varied incrementally. This rotates the nascent $p$ orbital on $\mathrm{C} \alpha$ from being perpendicular $\left(0^{\circ}\right.$ and $\left.180^{\circ}\right)$ to being parallel $\left(90^{\circ}\right)$ to the orbitals of the conjugated $\pi$ system of the Schiff base and pyridine ring. The curves fitted to the data $\operatorname{are} \cos ^{2}$ functions.

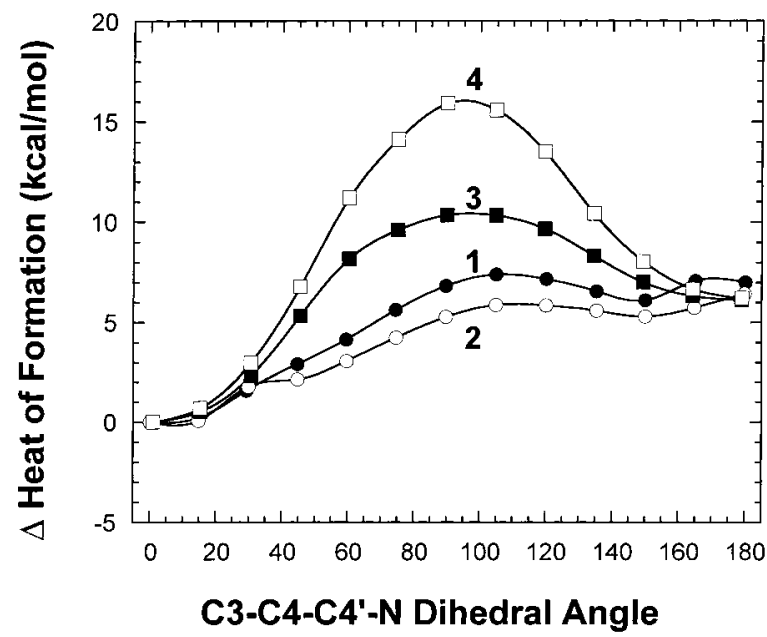

FIGURE 3: PM3 coordinate driving calculations to determine the contribution of the pyridine ring to the resonance stabilization of the transition state, and its geometric dependence. These calculations are similar to those reported in Figure 2 except that the $\mathrm{C} 3-\mathrm{C} 4-$ $\mathrm{C}^{\prime}-\mathrm{N}$ dihedral angle was varied.

structure. A total of 1000 structures were carried through 1000 generations, using molecular mechanics to optimize each. The lowest energy structure obtained from this calculation was very close to that originally built by eye, with the AIB carboxylate group interacting with Lys272, Gln52, and Arg406 (subsite A; Figure 4B). A similar Monte Carlo calculation was performed on the active-site model in which the $\epsilon$-amino group of Lys 272 was unprotonated, and yielded a structure in which the AIB carboxylate group interacts with Arg406 in a bidentate hydrogen bond/salt bridge (subsite B; Figure 4B).

The optimizations with the simple aldimine model were terminated when changes in the heat of formation were less than $0.0001 \mathrm{kcal} / \mathrm{mol} /$ iteration, with other convergence criteria kept at default values. The SM3 water model of Cramer and Truhlar (40) was employed in the solvent calculations, which were full optimizations rather than single point solvation energies.

The calculations with the active-site model required less aggressive pseudodiagonalization in the SCF calculations, through the use of the GRADUAL keyword. The activesite optimizations were terminated when changes in the heat of formation were less than $0.001 \mathrm{kcal} / \mathrm{mol} /$ iteration, with other convergence criteria kept at default values. Molecular mechanical correction to amide bonds (necessary to maintain planarity) was employed in all calculations on the active site model. All unmentioned parameters were kept at their default values.

Constrained minimization ("coordinate driving”) calculations were used to obtain the series of structures reported here. The reaction coordinate for decarboxylation of both the simple aldimine models and the active site models was associated with the $\mathrm{C} \alpha-\mathrm{CO}_{2}^{-}$bond length (Scheme 1). Decarboxylation reaction paths were calculated via a series of geometry optimizations in which the $\mathrm{C} \alpha-\mathrm{CO}_{2}{ }^{-}$bond length was fixed at small increments $(0.05 \AA)$ and the remainder of the structure was energy minimized. For each $\mathrm{C} \alpha-\mathrm{CO}_{2}{ }^{-}$bond length increment, the final structure from the previous step was used as the starting structure. Similarly, the variations in heats of formation with dihedral angles were obtained by energy minimization of the remainder of the structures at various fixed values of the dihedral angles.

The calculations of interaction energy vs $\mathrm{C} \alpha-\mathrm{CO}_{2}{ }^{-}$bond length for the active-site models were accomplished by separating into two distinct coordinate files the coenzymesubstrate component from the protein component using INSIGHTII (Biosym Technologies, Inc.). This was repeated for each of the 40 structures in the reaction path calculation. The individual PM3 heats of formation of the separated coenzyme-substrate and protein structures were calculated in SPARTAN.

\section{RESULTS AND DISCUSSION}

It is well documented that semiempirical methods give artificially large activation enthalpies, especially for proton transfers $(25,26)$. Modification of standard semiempirical parameter sets for specific reactions can lead to accurate reproduction of activation enthalpies $(27,28)$. Although this was not performed here, the relative magnitudes of the various effects presented below (e.g., stereoelectronic vs electrostatic) should be realistic.

(A) Simple Aldimine Model. (A.1) Reaction Path Calculations for Prototropic Isomers. Four prototropic isomers of PLP and its analogues interacting with a carboxylic acid at the pyridine nitrogen are shown in Scheme 2. Biochemists generally assume $\mathbf{2}$ to be the active form of the coenzyme in enzyme-catalyzed reactions. This assumption is based primarily on studies from Bruice's laboratory (29) in which it was demonstrated that the pyridine nitrogen protonated form of the Schiff base between 3-hydroxypyridine-4aldehyde and alanine transaminates more rapidly than the unprotonated form. Surprisingly, the effect of pyridine nitrogen protonation is only 75 -fold with acetate as buffer, and $N$-methylation to quaternize the pyridine ring of 3-hydroxypyridine-4-aldehyde increases the rate of transamination only 20 -fold (30).

Both gas phase and water model decarboxylation reaction path calculations were performed for the isomeric aldimine 

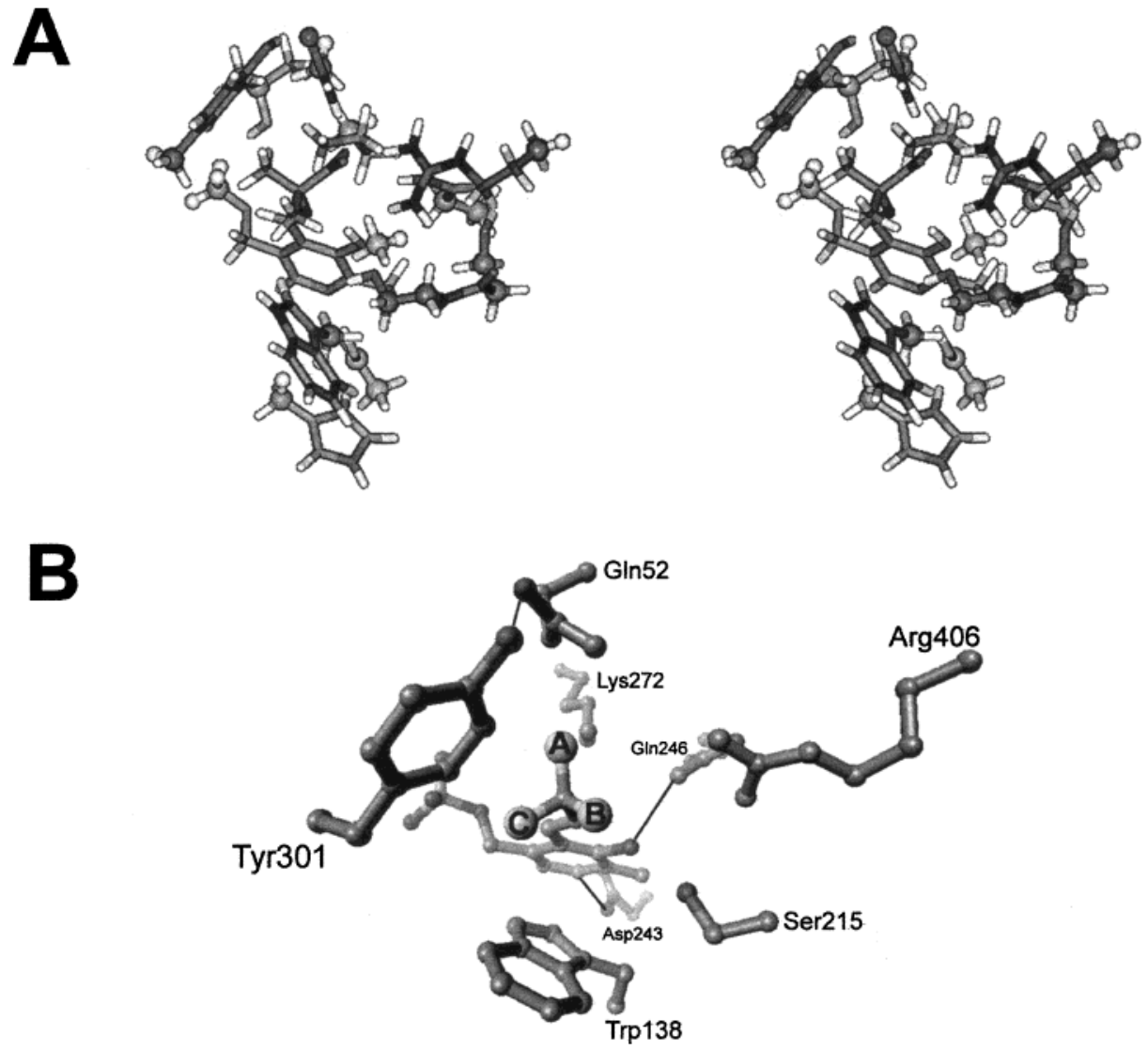

FIGURE 4: (A) Active site model of dialkylglycine decarboxylase. Generally, the active site residues were truncated to the C $\beta$ 's and the $\mathrm{C} \alpha$ 's replaced with hydrogens. The atoms that were held fixed during all calculations are shown as spheres. (B) Active-site diagram indicating the position of the binding subsites (A, B, and C).

Scheme 2

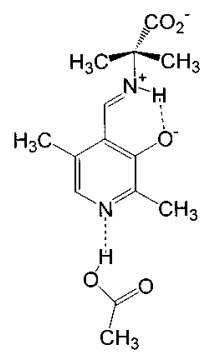

1<smiles></smiles>

3

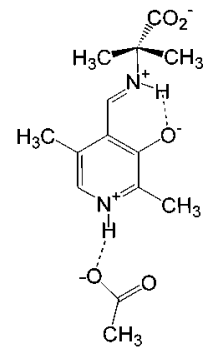

2

4<smiles>CC(=O)OON1C=C(C)C(C=NC2(C)CO2)C(O)=C1C</smiles>

models shown in Scheme 2. The results are shown in Figure 1. In both gas phase and water model calculations, the ground states for the isomers with a protonated pyridine nitrogen ( 2 and 4) are higher in energy than for the unprotonated isomers: $\Delta \Delta H \approx 14 \mathrm{kcal} / \mathrm{mol}$ for 1 vs 2 , and $\Delta \Delta H \approx 18$ $\mathrm{kcal} / \mathrm{mol}$ for $\mathbf{3}$ vs $\mathbf{4}$ for either the gas phase or water. The activation enthalpies for the pyridine nitrogen-protonated isomers are lower than for the unprotonated isomers: $\Delta \Delta H$ $\approx 4 \mathrm{kcal} / \mathrm{mol}$ in the gas phase and $\Delta \Delta H \approx 3.5 \mathrm{kcal} / \mathrm{mol}$ in the water model. This is expected if the unshaired pair of electrons developing on $\mathrm{C} \alpha$ in the transition state are stabilized by at least partial delocalization into the pyridine ring, as was originally proposed by Metzler et al. (31).

The activation enthalpies for the gas-phase calculations are low, ranging from $\sim 3.4 \mathrm{kcal} / \mathrm{mol}$ for the pyridine nitrogen protonated isomers $(\mathbf{2}, \mathbf{4})$ to $\sim 7.3 \mathrm{kcal} / \mathrm{mol}$ for the unprotonated isomers $(\mathbf{1}, \mathbf{3})$, as expected for decarboxylation of a naked carboxylate to give a highly resonance stabilized carbanion in a low dielectric medium. The activation enthalpies with the water model are much higher, ranging from 18 (2) to 28 (3) kcal/mol, as expected for strong groundstate solvation of the carboxylate ion. The results with the water model are in general agreement with the experimental free energy of activation of $25.3 \mathrm{kcal} / \mathrm{mol}$ at $25{ }^{\circ} \mathrm{C}$ for decarboxylation of the AIB-PLP aldimine in acetate buffer, pH 5.0 [R. F. Zabinski and M. D. Toney (2001) J. Am. Chem. Soc. (in press)].

(A.2) Stereoelectronic Effects. Dunathan (32) proposed in 1966 that PLP dependent enzymes can control reaction specificity by orienting, through specific binding interactions in the external aldimine intermediate, the bond to be broken such that it is parallel to the $p$ orbitals of the conjugated $\pi$ system. Stereoelectronic effects are well documented in organic chemistry (33). Here, they have a simple origin in the geometric dependence of $p-p$ orbital interactions, which follow a $\cos ^{2}$ function. In enzymatic reactions, experimental 
evidence for stereoelectronic effects has been sparse and measurements of their magnitudes not yet made.

Figure 2 presents data that quantitate the stereoelectronic effect in the decarboxylation reaction considered here. Transition state structures were obtained by stationary points optimizations on the highest energy structures from Figure 1. The transition state $\mathrm{C \alpha}-\mathrm{CO}_{2}^{-}$bond length for each tautomer was fixed, and constrained minimization performed at various $\mathrm{C} 4^{\prime}-\mathrm{N}-\mathrm{C} \alpha-\mathrm{CO}_{2}{ }^{-}$dihedral angles. As seen in Figure 2, the dependence of energy on the angle between the orbitals largely conforms to a $\cos ^{2}$ function, the discrepancy at least partially due to the asymmetry of the molecules. The lowest energy structure in each case occurs with this dihedral angle close to $90^{\circ}$ (orbital-orbital angle $\approx 0^{\circ}$ ), where the greatest orbital overlap occurs. The two enolimine isomers $(\mathbf{3}, \mathbf{4})$ show the greatest dependence of energy on dihedral angle since resonance stabilization through the $\pi$ system, as opposed to electrostatic ylide-type stabilization in the ketoenamine isomers $(\mathbf{1}, \mathbf{2})$, is dominant.

(A.3) Separation of Pyridine Ring and Aldimine Resonance Effects. The resonance stabilization quantitated above can be divided into two components: that from the Schiff base and that from the pyridine ring. Their relative contributions are obtained by combining the calculations shown in Figures 2 and 3. In Figure 3, the results of constrained minimizations in which the $\mathrm{C} 3-\mathrm{C} 4-\mathrm{C} 4{ }^{\prime}-\mathrm{N}$ dihedral angles of the transition state structures (with fixed $\mathrm{C} \alpha-\mathrm{CO}_{2}{ }^{-}$bond lengths) were varied are shown. The effect of loss of conjugation with the protonated pyridine ring is larger than with the unprotonated ring. It is largest in both cases when this dihedral angle is $\sim 90^{\circ}$, i.e., where the $\mathrm{C} 4$ and $\mathrm{C}^{\prime} p$ orbitals are orthogonal to each other. The difference in energy between the 0 and $180^{\circ}$ structures is largely due to the loss of the hydrogen bond between the aldimine nitrogen and O3'.

The data in Figure 2 report on loss of conjugation with both the Schiff base and the pyridine ring, while the data in Figure 3 report on loss of conjugation only with the pyridine ring. Thus, the maximal effects for $\mathbf{3}$ and $\mathbf{4}$, for example, in Figure 3 (4.3 and $9.8 \mathrm{kcal} / \mathrm{mol}$, respectively, as measured between 90 and $180^{\circ}$ to eliminate the $\mathrm{N}-\mathrm{O}^{\prime}$ hydrogen bonding contribution) and in Figure 2 (19.2 and $26.9 \mathrm{kcal} /$ mol, respectively) allow one to estimate the individual contributions of the unprotonated Schiff base and the unprotonated and protonated pyridine rings to the resonance effect: the unprotonated pyridine ring contributes $-4.3 \mathrm{kcal} /$ mol to the stabilization of (the transition state for) $\mathbf{3}$, and the protonated pyridine ring contributes $-9.8 \mathrm{kcal} / \mathrm{mol}$ to the stabilization of (the transition state for) 4. Remarkably, the contribution from the unprotonated Schiff base is dominant and relatively equal for both cases: $14.9 \mathrm{kcal} / \mathrm{mol}$ for (the transition state for) $\mathbf{3}$ and $17.1 \mathrm{kcal} / \mathrm{mol}$ for (the transition state for) 4 .

This striking result suggests that the simple pyruvoyl group of pyruvoyl dependent decarboxylases (34) should capture the lion's share of the catalytic power that pyridoxal phosphate has to offer. Accordingly, these two fundamentally different groups of decarboxylases share similar catalytic power. A major difference between these prosthetic groups may be that the 3'-hydroxyl group of PLP plays an important catalytic role in Schiff base formation (35). Bach et al. have reached essentially the same conclusion based on high-level

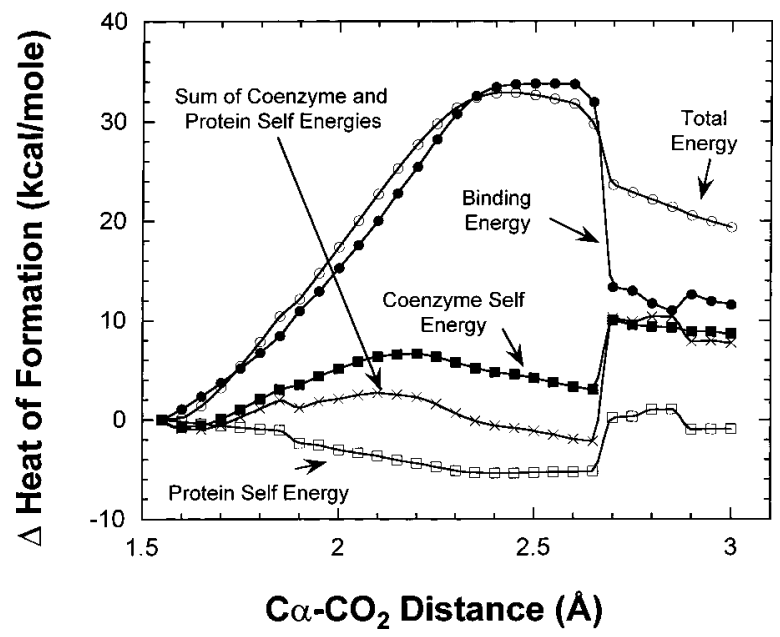

FIGURE 5: PM3 constrained energy minimization calculation for 3 in which the $\mathrm{C} \alpha-\mathrm{CO}_{2}{ }^{-}$bond length was lengthened, and deconvolution of the energy contributions to the total energy. The "total energy" is the calculated heat of formation for the active site model as a whole. The "coenzyme self-energy" is the heat of formation of the coenzyme-substrate adduct in isolation from the protein portion of the model. The "protein self-energy" is the heat of formation of the protein portion of the model in isolation from the coenzyme-substrate adduct. The "binding energy" is the difference between the "total energy" and the sum of the two "self-energies". This represents the energy of noncovalent interaction (electrostatic/ hydrogen bonding) between the coenzyme-substrate adduct and the protein portion of the model as a function of the reaction coordinate.

calculations on decarboxylation models in which the carboxyl group is protonated in the reactant state $(36,37)$.

(B) Dialkylglycine Decarboxylase. Dialkylglycine decarboxylase is an unusual PLP dependent enzyme that catalyzes the oxidative decarboxylation of AIB in the first half-reaction and the transamination of pyruvate to L-alanine in the second of the ping-pong kinetic mechanism (38).

(B.1) Active-Site Model. The active site model obtained from crystallographic coordinates and Monte Carlo calculations was used first in a series of constrained minimization calculations in which the $\mathrm{C} 4^{\prime}-\mathrm{N}-\mathrm{C} \alpha-\mathrm{CO}_{2}{ }^{-}$dihedral angle was varied with the carboxylate initially placed in either binding subsite A or B (Figure 4B). These calculations (data not shown) show that the model with Lys 272 protonated prefers the carboxylate in subsite A and that with Lys272 unprotonated prefers it in subsite B. This result is expected based on the requirement for unprotonated Lys 272 to act as a general base catalyst in the alanine transamination halfreaction, in which the $\mathrm{C}-\mathrm{H}$ bond is made/broken from subsite A and the carboxylate group is in subsite B. Optimizations of ground-state geometries for the four prototropic isomers (where Asp243 replaces acetate in Scheme 2 ) show that $\mathbf{3}$ is the most stable in the active site, as found in both the gas phase and water with the aldimine model above.

(B.2) Reaction Path Calculations. Decarboxylation reaction path calculations were performed for the four prototropic isomers of the active-site model. The energy profiles for all show abrupt changes in energy at points along the reaction paths. Figure 5 shows the results obtained with $\mathbf{3}$. The sudden energy drop at $2.7 \AA$ corresponds structurally to movement of the Lys $272 \epsilon-\mathrm{NH}_{3}{ }^{+}$group to within van der Waals distance of $\mathrm{C}^{\prime}$. Reverse calculations starting from $\mathrm{C} \alpha-\mathrm{CO}_{2}{ }^{-}$bond 


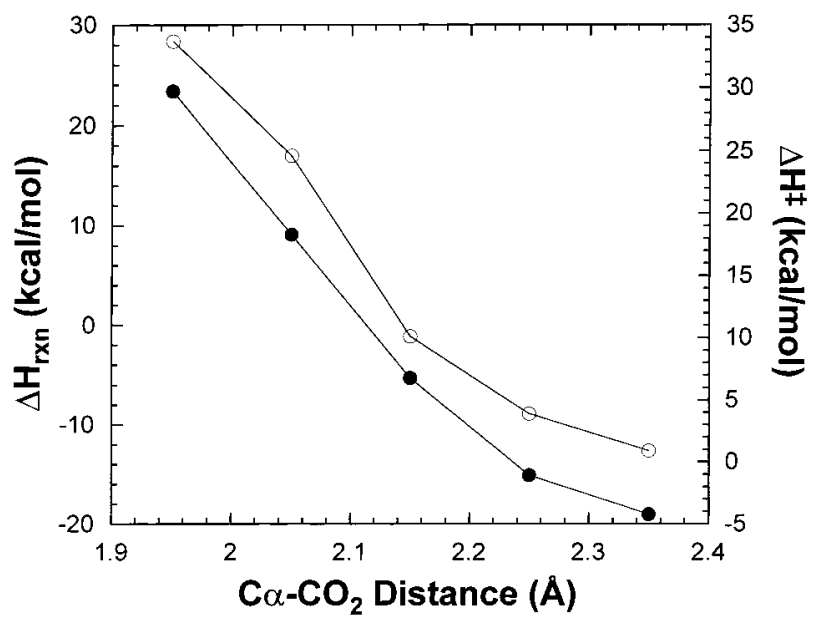

FIGURE 6: PM3 energies for proton transfer (solid circles) and the barrier to proton transfer (open circles) from the $\epsilon-\mathrm{NH}_{3}{ }^{+}$group of Lys272 to $\mathrm{C}^{\prime}{ }^{\prime}$ as a function of $\mathrm{C} \alpha-\mathrm{CO}_{2}{ }^{-}$bond length for 3 .

lengths of $3.5 \AA$ do not yield identical series of structures. These results show that the reaction coordinate cannot be simply associated with the $\mathrm{C} \alpha-\mathrm{CO}_{2}{ }^{-}$bond length, but contains another component(s).

The movement of Lys272 to within van der Waals distance of $\mathrm{C}^{\prime}$ at a $\mathrm{C} \alpha-\mathrm{CO}_{2}{ }^{-}$bond length of $2.7 \AA$ suggests that a proton transfer from Lys 272 to $\mathrm{C}^{\prime}$, , simultaneous with $\mathrm{C} \alpha-$ $\mathrm{CO}_{2}{ }^{-}$bond breaking, may be the missing component of the reaction coordinate. The energy deconvolution presented in Figure 5 shows that a sharp decrease in the energy of interaction (binding energy) between the protein and coenzyme-substrate components at $2.7 \AA$ drives the sudden structural change. The known overestimation of proton transfer barriers with PM3 is likely to responsible for the observed structural change instead of a proton transfer, even though proton transfer is thermodynamically favorable.

(B.3) Proposed Concerted Transition State. The magnitude of the energy barrier and thermodynamic driving force for proton transfer from Lys $272 \epsilon-\mathrm{NH}_{3}{ }^{+}$to the coenzyme C4' in 3 were calculated via constrained minimizations for structures in which the $\mathrm{C} \alpha-\mathrm{CO}_{2}^{-}$bond length is between 1.95 and $2.35 \AA$. A plot of the calculated energy barriers and the energy differences is presented in Figure 6. These calculations show that at values of $\mathrm{C} \alpha-\mathrm{CO}_{2}{ }^{-}$bond length $>2.1 \AA$ the transfer of a proton from the $\epsilon-\mathrm{NH}_{3}{ }^{+}$of Lys 272 to $\mathrm{C}^{\prime}{ }^{\prime}$ is favorable. The calculated barriers to this proton transfer are small given the known overestimation of protontransfer barriers by PM3, and are almost nonexistent at $\mathrm{C} \alpha-$ $\mathrm{CO}_{2}^{-}$bond lengths $>2.35 \AA$ A. Qualitatively similar results are obtained with $\mathbf{2}$ and $\mathbf{4}$, although the proton-transfer energy barriers are higher and driving forces lower.

These calculations suggest that proton transfer from Lys272 to $\mathrm{C}^{\prime}$ ' is the missing component of the complex reaction coordinate, implying that a concerted decarboxylation/proton transfer in which $\mathrm{C} \alpha-\mathrm{CO}_{2}{ }^{-}$bond breaking leads proton transfer to $\mathrm{C}^{\prime}$ ' is a likely mechanistic alternative with this enzyme. Experimental evidence supporting this same conclusion was presented by Zhou et al. (24).

Previously, Sun et al. (39) provided experimental evidence that less than 1 in $10^{5}$ turnovers of AIB occurs with protonation on $\mathrm{C} \alpha$, with $\mathrm{C}^{\prime}$ ' protonation the major pathway. The concerted transition state proposed here is important in that it provides a simple explanation for the experimentally observed high specificity of DGD for oxidative decarboxylation (i.e., protonation on $\mathrm{C}^{\prime}$ ' vs $\mathrm{C} \alpha$ ) of $\mathrm{AIB}$ and other 2,2dialkylglycines. Computational studies with more sophisticated electronic structure methods are currently underway to validate the present PM3-based conclusions with methods that do not suffer from overestimation of proton-transfer barriers.

\section{REFERENCES}

1. Toney, M. D., Hohenester, E., Cowan, S. W., and Jansonius, J. N. (1993) Science 261, 756.

2. Hohenester, E., Keller, J. W., and Jansonius, J. N. (1994) Biochemistry 33, 13561.

3. Toney, M. D., Hohenester, E., Keller, J. W., and Jansonius, J. N. (1995) J. Mol. Biol. 245, 151.

4. Malashkevich, V. M., Strop, P., Keller, J. W., Jansonius, J. N., and Toney, M. D. (1999) J. Mol. Biol. 294, 193.

5. Marlier, J. F., and O'Leary, M. H. (1986) J. Am. Chem. Soc. 108, 4896, and references therein.

6. Austin, J. C., Zhao, Q., Jordan, T., Talalay, P., Mildvan, A. S., and Spiro, T. G. (1995) Biochemistry 34, 4441.

7. Callender, R., and Deng, H. (1994) Annu. Rev. Biophys. Biomol. Struct. 23, 215.

8. Chen, Y. Q., Kraut, J., Blakley, R. L., and Callender, R. (1994) Biochemistry 33, 7021.

9. Deng, H., Chan, A. W., Bagdassarian, C. K., Estupinan, B., Ganem, B., Callender, R. H., and Schramm, V. L. (1996) Biochemistry 35, 6037.

10. Deng, H., Kurz, L. C., Rudolph, F. B., and Callender, R. (1998) Biochemistry 37, 4968.

11. Dong, J., Xiang, H., Luo, L., Dunaway-Mariano, D., and Carey, P. R. (1999) Biochemistry 38, 4198.

12. Johnson, F. A., Lewis, S. D., and Shafer, J. A. (1981) Biochemistry 20, 52.

13. Lewis, S. D., Johnson, F. A., and Shafer, J. A. (1981) Biochemistry 20, 48.

14. Mackenzie, N. E., Malthouse, J. P., and Scott, A. I. (1984) Science 225, 883.

15. Perez-Canadillas, J. M., Campos-Olivas, R., Lacadena, J., Martinez del Pozo, A., Gavilanes, J. G., Santoro, J., Rico, M., and Bruix, M. (1998) Biochemistry 37, 15865.

16. Sanchez-Ruiz, J. M., Iriarte, A., and Martinez-Carrion, M. (1991) Arch. Biochem. Biophys. 286, 38.

17. Schnackerz, K. D., and Mozzarelli, A. (1998) J. Biol. Chem. 273, 33247.

18. Stirtan, W. G., and Withers, S. G. (1996) Biochemistry 35, 15057.

19. Gallagher, T., Snell, E. E., and Hackert, M. L. (1989) J. Biol. Chem. 264, 12737.

20. Ashley, J. A., Lo, C.-H. L., McElhaney, G. P., Wirsching, P., and Janda, K. D. (1993) J. Am. Chem. Soc. 115, 2515.

21. Mulholland, A. J., Grant, G. H., and Richards, W. G. (1993) Protein Eng. 133.

22. Cunningham, M. A., and Bash, P. A. (1997) Biochimie 79, 687.

23. Gao, J. (1996) Acc. Chem. Res. 29, 298.

24. Zhou, X., Jin, X., Medhekar, R., Chen, X., Dieckmann, T., and Toney, M. D. (2001) Biochemistry 40, XXXX-XXXX.

25. Kallies, B., and Mitzner, R. (1995) J. Mol. Model. 1, 68.

26. Zheng, Y.-J., Merz, K. M., and Farber, G. K. (1993) Protein Eng. 6, 479.

27. Kim, Y. (1996) J. Am. Chem. Soc. 118, 1522.

28. Alhambra, C., Wu, L., Zhang, Z.-Y., and Gao, J. (1998) J. Am. Chem. Soc. 120, 3858.

29. Auld, D. S., and Bruice, T. C. (1967) J. Am. Chem. Soc. 89, 2098.

30. Maley, J. R., and Bruice, T. C. (1970) Arch. Biochem. Biophys. 136, 187

31. Metzler, D. E., Ikawa, M., and Snell, E. E. (1954) J. Am. Chem. Soc. $76,648$. 
32. Dunathan, H. C. (1966) Proc. Natl. Acad. Sci U.S.A. 55, 712.

33. Deslongchamps, P. (1983) Stereoelectronic effects in organic chemistry, Pergamon, New York.

34. van Poelje, P. D., and Snell, E. E. (1990) Annu. Rev. Biochem. $59,29$.

35. French, T. C., Auld, D. S., and Bruice, T. C. (1965) Biochemistry 4, 77.

36. Bach, R. D., and Canepa, C. (1997) J. Am. Chem. Soc. 119, 11725.
37. Bach, R. D., Canepa, C., and Glukhovtsev, M. N. (1999) J. Am. Chem. Soc. 121, 6542.

38. Bailey, G. B., and Dempsey, W. B. (1967) Biochemistry 6, 1526.

39. Sun, S., Zabinski, R. F., and Toney, M. D. (1998) Biochemistry 37,3865

40. Cramer, C. J., and Truhlar, D. G. (1992) J. Comput. Chem. 13, 1089-1097.

BI0012383 